Submitted: 8.9.2017

Accepted: 28.11.2017

Conflict of interest

None.
DOI: $10.1111 / d d g .13529$

\section{Vertical transmission of herpes simplex virus: an update}

Anil Kumar Bhatta', Uma Keyal', Yeqiang Liu',

\section{Q1 Emese Gellen²}

(1) Department of Dermatopathology Shanghai Skin Disease Hospital, Tongji University School of Medicine, Shanghai, China

(2) Department of Dermatology Faculty of Medicine University of Debrecen, Hungary

\begin{abstract}
Summary
Herpes simplex virus (HSV)-1 and -2 infections are highly prevalent worldwide. HSV infection during pregnancy can result in neonatal herpes infection, which is characterized by lifelong infection with periods of latency and reactivation. HSV can be acquired by an infant during one of three periods: in utero (5\%), peripartum (85\%), or postnatal (10 \%). Neonatal HSV is a rare but significant infection that may be associated with severe morbidity and mortality, especially if there is dissemination or central nervous system involvement. Diagnostic and therapeutic advances have led to a reduction in mortality and, to a lesser extent, improvement of neurodevelopmental outcomes, but further developments are still needed. It is essential to improve the clinician's ability to identify infants who are at increased risk of HSV infection and to prevent mother-to-child transmission. The development of novel antiviral agents with higher efficacy is a worthwhile aim for the future.
\end{abstract}

\section{Introduction}

Herpes simplex virus (HSV) is one of the most common sexually transmitted pathogens and is responsible for genital herpes infection. Both HSV-1 and HSV-2 can cause genital herpes as wells as neonatal infection via mother-to-child transmission, which can lead to serious health problems for the newborn [1]. Maternal infection with HSV before or during childbirth can cause neonatal infection through vertical transmission or close contact. Neonatal HSV infection refers to infection within 28 days of the neonatal period, including intrauterine infection (also known as innate infection), birth canal infection, and postnatal infection $[2,3]$. In order to prevent and better control HSV infection of newborns, it is necessary to fully understand the prevalence, transmission mechanisms, influencing factors as well as prevention and control measures of HSV mother-to-child transmission.

\section{Prevalence, incidence and burden of the disease}

Maternal infection with HSV is very common and is associated with lifelong infection in both developed and developing countries. The prevalence varies from country to country, which is well illustrated by the fact that in Nigeria $99.4 \%$ of pregnant women were seropositive for anti-HSV-1 and HSV-2 IgG antibodies, while in South Africa the seroprevalence of HSV-2 infection was $58.7 \%$. Ethiopia had the lowest rate of African countries, with $32.1 \%$ [4-6]. Similar to Ethiopia, the overall weighted prevalence of HSV-2 was $31.4 \%$ in Haiti, while in Korea, Kim et al. reported an HSV2 prevalence of $17 \%$ [7, 8]. In the United States, between 1989-2010, $53 \%$ of pregnant women were seropositive for HSV-1, $9 \%$ for HSV-2 and $15 \%$ were seropositive for both [9]. A decrease in HSV-1 and HSV-2 infections was reported in Finland, where the HSV-1 positivity rate of sera decreased 
from $69.5 \%$ to $45 \%$ over 20 years. The seroprevalence of HSV-2 decreased to a lesser extent, from $17.5 \%$ to $11 \%$ [10]. However, the authors found that $48 \%$ of women of childbearing age were at risk of primary HSV infection during pregnancy. In the Netherlands, there is also a tendency towards decreasing HSV-1 and HSV-2 seroprevalence. In 1995 , the positivity rate of HSV-1 sera was $47.7 \%$, while for HSV-2 it was $6.8 \%$. By 2006, seropositivity of HSV-1 had decreased to $42.7 \%$, while HSV-2 seropositivity was 6.0 \% [11]. In Germany, according to Korr et al., from 19971999 to 2008-2011 HSV-1 seroprevalence decreased from $82.1 \%$ to $78.4 \%$ while HSV-2 seroprevalence decreased from $13.3 \%$ to $9.6 \%$ (Table 1) [12]. This also means that an increasing number of adolescents lack protective antibodies against HSV-1 at the beginning of their sexual life [13]. However, HSV-1 still causes primarily genital herpes. The main risk factors for genital HSV infection are having two or more sexual partners, history of other sexually transmitted disease(s), female gender, use of contraceptives, low educational level, belonging to a minority ethnic group and sexual activity at an early age $[6,14,15]$.

In case of asymptomatic infection of the mother, the alarming clinical signs are missing, so that it is difficult to recognize an HSV infection in newborns; this can lead to serious health consequences. In Australia, the incidence of
HSV infection in newborns was 3.27/100,000 live births. Of these, $62.7 \%$ were caused by HSV-1 and $37.2 \%$ by HSV-2 [16]. In the United States in 2006, the incidence of neonatal herpes was 9.6/100,000 live births [17], but among Malawian female adolescents the incidence was much higher, 71.8/100,000 live births [18]. According to Hemelaar et al., the incidence of neonatal herpes infections in the Netherlands was $4.7 / 100,000$ births for the period 2006-2011, which was twice as high as the incidences reported in previous studies [19]. In one of the most recent studies [20], the estimated global incidence of neonatal herpes infection was 10.3/100,000 live births, with the largest number of cases in Africa, accounting for $37 \%$ of the global number of cases. However, the exact incidence is still not clear due to the lack of data on neonatal HSV infection (Table 2).

The mortality rate of neonatal herpes is high, even after intravenous acyclovir treatment, imposing a heavy burden on both the family and society. Treating neonatal herpes is expensive, and involves the costs of hospital stay, intensive care, intravenous drug therapy, laboratory tests, and the long-term costs of disability in case of severe neurological outcomes. According to Ambroggio et al., the cost of hospitalization was up to $\$ 37,431$ per infant (quartile spacing: $\$ 14,667$ to $\$ 74,519)[21]$.

Table 1 Prevalence of $\mathrm{HSV}_{1} \& \mathrm{HSV}_{2}$

\begin{tabular}{|c|c|c|c|c|}
\hline Year & $\mathrm{HSV}_{1} / \mathrm{HSV}_{2}$ & Country & $\%$ seroprevalence & Reference \\
\hline- & $\mathrm{HSV}_{1} \& \mathrm{HSV}_{2}$ & Nigeria & 99.4 & [4-6] \\
\hline- & $\mathrm{HSV}_{2}$ & South Africa & 58.7 & [4-6] \\
\hline- & $\mathrm{HSV}_{2}$ & Ethiopia & 32.1 & {$[4-6]$} \\
\hline- & $\mathrm{HSV}_{2}$ & Haiti & 31.4 & {$[7,8]$} \\
\hline- & $\mathrm{HSV}_{2}$ & Korea & 17 & {$[7,8]$} \\
\hline 1989-2010 & $\mathrm{HSV}_{1}$ & US & 53 & [9] \\
\hline 1989-2010 & $\mathrm{HSV}_{2}$ & US & 9 & [9] \\
\hline $1989-2010$ & $\mathrm{HSV}_{1} \& \mathrm{HSV}_{2}$ & US & 15 & [9] \\
\hline 1995 & $\mathrm{HSV}_{1}$ & Netherlands & $47 \cdot 7$ & [11] \\
\hline 1995 & $\mathrm{HSV}_{2}$ & Netherlands & 6.8 & [11] \\
\hline 2006 & $\mathrm{HSV}_{1}$ & Netherlands & 42.7 & [11] \\
\hline 2006 & $\mathrm{HSV}_{2}$ & Netherlands & 6 & [11] \\
\hline 1997-1999 & $\mathrm{HSV}_{1}$ & Germany & 82.1 & [12] \\
\hline $2008-2011$ & $\mathrm{HSV}_{1}$ & Germany & 78.4 & [12] \\
\hline 1997-1999 & $\mathrm{HSV}_{2}$ & Germany & 13.3 & [12] \\
\hline $2008-2011$ & $\mathrm{HSV}_{2}$ & Germany & 9.6 & [12] \\
\hline- , not available. & & & & \\
\hline
\end{tabular}


Table 2 Incidence of neonatal herpes.

\begin{tabular}{|c|c|c|c|c|}
\hline Year & Country & Herpes simplex & $\begin{array}{l}\text { Incidence (per 100,000) } \\
\text { live births }\end{array}$ & Reference \\
\hline 2006 & USA & Neonatal herpes & 9.6 & [17] \\
\hline 2010 & Malawi & Neonatal herpes & 71.8 & {$[18]$} \\
\hline 2006-2011 & Netherlands & Neonatal herpes & 4.7 & [19] \\
\hline $2010-2015$ & World & Neonatal herpes & 10.3 Africa highest ( $37 \%$ ) & {$[20]$} \\
\hline
\end{tabular}

\section{Clinical types of HSV infection}

HSV-1 is predominantly associated with orolabial infection, but may be responsible for genital herpes as well. HSV-2 is almost exclusively sexually transmitted, causing genital herpes. However, the number of genital herpes infections triggered by HSV-1 has increased during the past years [22]. Moreover, Brown et al. reported that HSV-1 had a higher risk of mother-to-child transmission than HSV-2 [23]. Primary HSV infections in women are mainly asymptomatic, but can present as painful erythematous papules, followed by vesicles and finally erosions on the vulva, labia major and minor, vagina and cervix, lasting for approximately $8-10$ days. Recurrent herpes may be asymptomatic or symptomatic.

\section{Types of mother-to-child transmission and main risk factors for neonatal herpes infection}

HSV mother-to-child transmission can take place in the uterus (in utero transmission), during delivery (peripartum neonatal transmission) and after birth (postnatal HSV infection) $[1,24] .85 \%$ of mother-to-child HSV transmissions occur during the delivery stage, when there is viral shedding from the genital tract - particularly in secretions from the vagina. During pregnancy, both hematogenous spread and vertical transmission can lead to placental or amniotic membrane involvement, resulting in intrauterine (congenital, antepartum) HSV transmission, which accounts for $5 \%$ of genital HSV infections. HSV can also be transmitted postnatally to the newborn, comprising $10 \%$ of cases. Postpartum infections occur via direct contact of the newborn with HSV-infected persons, mainly from the mother by kissing, usually from her orolabial or cutaneous lesions. However, $70 \%$ of mothers with HSV-infected newborns have no history, symptoms or signs of HSV infection at delivery [25]. A possible explanation is that recurrent genital herpes during pregnancy is more common than primary genital herpes, and recurrent genital herpes is often asymptomatic. According to Straface et al., nearly half of HSV infections in newborns were caused by the mother's recurrent genital herpes infection [26].

During pregnancy, the risk of transmission of primary genital herpes is higher than that of recurrent genital herpes. $30 \%$ to $50 \%$ of newborns are infected with HSV if the mother has a primary HSV infection in the third trimester [27]. In this case, there is not enough time for complete seroconversion to IgG before delivery and the infant is born without any protective passive $\mathrm{Ig} G$ from the mother. The risk of neonatal infection is therefore highest at the time of the delivery. If a pregnant woman has primary HSV infection in the first trimester, her transmission efficiency is low; even if herpetic lesions can be observed in the genital area, the risk of neonatal infection is less than $1 \%$ [2]. These data were confirmed by Brown et al., who found that of 177 HSV-positive and antibody-tested pregnancies, 26 pregnant women had primary genital herpes, and 151 had recurrent genital herpes at the time of delivery [28]. The results showed that the risk of neonatal herpes infection was 59.3 times as high for primary genital herpes than for recurrent genital herpes. Further analysis revealed that HSV transmission to newborns was due to primary genital herpes in $44.4 \%$ of the cases, and by recurrent infections in $23.5 \%$ of cases. The transmission rate of recurrent genital herpes from mother to child was only $1.3 \%$. Maternal HSV antibodies delivered to the fetus via the placenta have a protective effect. One major risk factor for HSV transmission from mother to child is the absence of maternal serum HSV antibody. The rupture time of the membrane is also closely related to transmission risk. Finger-Jardim et al. reported that if the time between rupture of membranes and delivery is more than six hours, the risk of retrograde infection from the cervix is approximately fourfold [29]. For mothers with active herpes at the time of delivery, the risk of neonatal HSV infection can be significantly reduced if cesarean section is performed within four hours after rupture. If the rupture time is more than four hours, regardless of the mode of delivery, almost all newborns will be infected with HSV. In case of genital herpes symptoms during pregnancy, obstetricians would probably recommend cesarean section as the preferred mode of delivery. Studies that have sought to determine whether cesarean section can 
really reduce the risk of neonatal HSV infection are limited, and most have not been properly conducted. According to some studies, cesarean section can reduce the risk of motherto-child HSV transmission [23, 30]. However, performing cesarean sections before membrane rupture cannot block the spread of HSV completely, due to the possibility of intrauterine transmission [31].

In addition to the main factors mentioned above, such as cervical HSV positivity, fetal skin integrity can also affect the efficiency of mother-to-child HSV transmission. Transmission is significantly higher if there is cervical HSV positivity. At the same time, invasive fetal monitoring and fetal examination can also increase the risk of infection [23].

\section{Clinical manifestations of neonatal HSV infection}

In case of in utero HSV transmission, a characteristic triad of cutaneous symptoms (vesicles, erosions, aplasia cutis, hyperor hypopigmentation, scarring), neurological manifestations (hydranencephaly, microcephaly, intracranial calcifications, meningoencephalitis) and ophthalmologic symptoms (microphthalmia, chorioretinitis, optic atrophy) can be observed. Both primary and recurrent maternal HSV infection can lead to in utero transmission of the virus $[32,33]$.

If administration of antiretroviral treatment is not timely in cases of neonatal herpes, the mortality rate is up to $60 \%$. Even cases of early use of acyclovir treatment may still result in serious disability [2].

According to clinical manifestations, neonatal HSV infections acquired in the peripartum or postpartum period can be divided into three categories: the first is when the disease is confined to the skin, eyes and lips (skin, eye, and/or mouth, referred to as SEM); the second is central nervous system infection and the third is disseminated infection. The timing of the first appearance of symptoms, the characteristic findings, mortality rates and neurodevelopmental outcomes are summarized in Table 3. SEM disease is the mildest and accounts for $45 \%$ of HSV infection in newborns. The central nervous system and other organs are not affected. [34]. Without treatment, HSV can easily lead to central nervous system infection or disseminated disease. Central nervous system infection constitutes $30 \%$ of cases and only $65 \%$ of the newborns will have active cutaneous lesions. Cerebrospinal fluid examination will show mildly elevated white blood cells and a slight increase in protein [35, 36]. $25 \%$ of newborns have disseminated HSV infection. The infection can involve a variety of tissues and organs (such as brain, lung, liver, adrenal gland, skin, eyes and mouth), causing viral sepsis, respiratory failure, liver failure and disseminated intravascular coagulation (DIC), but only $60 \%$ of the infants have active cutaneous lesions [36]. Encephalitis is the most common complication, and fever is only present in a few cases [35].

\section{Diagnosis of genital herpes infection}

Both primary and recurrent herpes infections can be symptomatic (with prodromal symptoms) or asymptomatic. In case of clinical symptoms, not every patient will have typical cutaneous lesions (papules, vesicles and erosions) with local lymphadenopathy. Laboratory tests should therefore be performed if a genital herpes infection is suspected.

Table 3 Clinical manifestations of neonatal HSV infection.

\begin{tabular}{|c|c|c|c|}
\hline & $\begin{array}{l}\text { Skin, eye and mouth } \\
\text { (SEM) symptoms }\end{array}$ & $\begin{array}{l}\text { Central nervous system } \\
\text { infection }\end{array}$ & Disseminated disease \\
\hline First appearance of symptoms & 10-12 days after birth & 16-19 days after birth & 10-12 days after birth \\
\hline Characteristic symptoms & Conjunctivitis, vesicles & $\begin{array}{l}\text { Epilepsy, lethargy, } \\
\text { irritability, poor ap- } \\
\text { petite, unstable body } \\
\text { temperature }\end{array}$ & $\begin{array}{l}\text { Viral sepsis: respiratory failu- } \\
\text { re, liver failure, disseminated } \\
\text { intravascular coagulation }\end{array}$ \\
\hline \multicolumn{4}{|l|}{ Without intravenous acyclovir } \\
\hline 1-year mortality rate & - & $50 \%$ & $85 \%$ \\
\hline Rate of normal neurodevelopment & - & $33 \%$ & $50 \%$ \\
\hline \multicolumn{4}{|c|}{ Use of intravenous acyclovir $60 \mathrm{mg} / \mathrm{kg} /$ day } \\
\hline 1-year mortality rate & - & $4 \%$ & $29 \%$ \\
\hline Rate of normal neurodevelopment & - & $31 \%$ & $83 \%$ \\
\hline
\end{tabular}




\section{Laboratory examination}

To detect maternal infection, swabs should be taken from the mother's cervical and vaginal secretions or skin lesions. HSV DNA detection is now considered to be the gold standard for diagnosis. HSV DNA detection with PCR assays increases HSV detection rates significantly compared with virus culture, and the conditions for sample storage and transport are less stringent. If determination of anti-viral sensitivity is needed, cell culture should also be performed. In case of primary genital herpes, HSV typing should be done as well. Viral antigen detection methods are no longer recommended [37]. Type-specific serological tests can be used for detecting and monitoring HSV infection in pregnancy [38].

To confirm neonatal infection, isolation of HSV by culture is still the gold standard diagnostic method. Swabs should be taken 12-24 hours after birth from skin lesions, conjunctiva, mouth, nasopharynx and rectum [39].

Detection of HSV DNA from neonatal blood with PCR can confirm herpes simplex virus infection. In suspected central nervous system infection, cerebrospinal fluid (CSF) specimens can be cultured, or HSV DNA can be detected in neonatal CSF. The sensitivity of HSV DNA detection in CSF is higher than the sensitivity of virus culture; it is also suitable for HSV typing. It is therefore the preferred laboratory diagnostic method in cases of central nervous system infection [38].

Neonatal serum alanine aminotransferase (ALT) detection can be used in disseminated neonatal infection. It should be checked within 24 hours of birth; if ALT is twice as high as the upper limit of the normal range, neonatal disseminated infection can be suspected [24].

\section{Treatment of maternal and neonatal herpes infections and prognosis of neonatal herpes infection}

\section{First episode of genital herpes}

Primary genital herpes can cause severe genital ulcerations and neurologic involvement; every patient with first-episode genital herpes should therefore be treated. Pregnant women should be given intravenous acyclovir if they are severely infected, but if they have positive HSV serology without a history of genital herpes, antiviral therapy is not recommended.

According to the latest treatment guidelines (2016) of the World Health Organization (WHO), the recommended regime for the first episode of genital herpes is as follows [37, 40]:

- oral acyclovir $400 \mathrm{mg}, 3$ times a day, for 10 days; or

- oral acyclovir $200 \mathrm{mg}, 5$ times a day for 10 days; or

- oral valacyclovir $500 \mathrm{mg}$ twice daily for 10 days; or

- oral famciclovir $250 \mathrm{mg} 3$ times daily for 10 days.

\section{Recurrent genital herpes}

Genital herpes recurrences usually cause minor symptoms, therefore decisions about therapy should be discussed with the patient. Oral acyclovir, famciclovir and valaciclovir can reduce the duration and severity of the symptoms. Treatment should be given within 24 hours of onset or in the prodromal phase.

WHO recommends [37, 40]:

- oral acyclovir $400 \mathrm{mg}, 3$ times a day for 5 days; or

- oral acyclovir $800 \mathrm{mg}$ twice daily for 5 days; or

- oral acyclovir $800 \mathrm{mg}, 3$ times a day for 2 days; or

- oral valacyclovir $500 \mathrm{mg}$ twice daily for 3 days; or

- oral famciclovir $250 \mathrm{mg}$ twice daily for 5 days.

Slight differences can be observed between the WHO guidelines and the latest recommendations of the US Centers for Disease Control and Prevention (CDC). The CDC recommends [37, 41]:

- oral acyclovir $400 \mathrm{mg}, 3$ times a day; or

- oral valacyclovir $500 \mathrm{mg}$ twice a day; taken from $36^{\text {th }}$ week of pregnancy.

For pregnant women with frequent or severe genital herpes during pregnancy, WHO recommends long-term inhibitory regimens $[37,40]$ :

- oral acyclovir $400 \mathrm{mg}$ twice daily or

- valacyclovir $500 \mathrm{mg}$ twice daily; or

- famciclovir $250 \mathrm{mg}$ twice daily for at least 6-12 months.

\section{Treatment of neonates}

In highly suspected or newly diagnosed neonatal HSV infections, immediate antiviral therapy should be administered. Early and effective antiviral therapy is the most critical factor affecting prognosis.

Treatment recommendations by the CDC are acyclovir $20 \mathrm{mg} / \mathrm{kg}$ i.v. every 8 hours for 14 days for disease limited to the skin and mucous membranes, or for 21 days for disseminated or central nervous system infection [41]. After 21 days of treatment, re-analysis of the cerebrospinal fluid is needed. If HSV DNA test of the CSF is negative, treatment should come to an end - but if the test result is positive, continuous intravenous infusions should be given until the test result is negative.

\section{Prognosis of neonates}

Comparison of low-dose therapy (i.v. acyclovir $30 \mathrm{mg} / \mathrm{kg}$ / day, every 8 hours) with high-dose acyclovir treatment (i.v. acyclovir $60 \mathrm{mg} / \mathrm{kg} /$ day every 8 hours) has shown that the higher dose can significantly improve the survival rate of 
neonates with HSV infection. With high-dose acyclovir treatment, Kimberlin et al. found a mortality rate at 24 months of $31 \%$ with disseminated disease and $6 \%$ with central nervous system infection [42]. With low-dose acyclovir, the mortality rate at 24 months was $61 \%$ with disseminated infection and $19 \%$ with central nervous system infection. High dose acyclovir can also reduce the incidence of severe HSV-related neurological sequelae. The probability of normal development at 12 months of age was 6.6 times higher with high-dose acyclovir than with lower doses. In the acute phase of neonatal HSV infection, oral acyclovir improves the prognosis of the disease. In central nervous system infection, intravenous acyclovir can reduce the occurrence of neurological sequelae, and the chance of normal development of the nervous system is higher. It is therefore recommended to give oral acyclovir at a dose of $300 \mathrm{mg}$ three times a day for six months after completion of intravenous therapy. However, long-term use of acyclovir may cause neutropenia in infants; the blood count should therefore be closely monitored during treatment, first at two weeks and then at four weeks after initiation of acyclovir. If the neutrophil count is normal, a monthly check is sufficient [43].

\section{Prevention: improve mother-to-child transmission strategy}

\section{Prevention of infection during pregnancy}

Pregnant women who are not infected with genital herpes should avoid sexual intercourse or genital contact during the third trimester with a partner who has known or suspected genital herpes. The preventive effect of antiviral therapy is unknown. There is no evidence that antiviral therapy reduces the risk of HSV infection of pregnant women, and it is not recommended for prevention.

The WHO and US CDC guidelines do not recommend routine HSV-2 serological screening for pregnant women. Although HSV vaccination may be the best way to prevent infection, it is still in the experimental stage. In the United States, population trials have been conducted to evaluate the effect of recombinant gD2 subunit vaccines. Recent studies have found that $\mathrm{gV} 2$ vaccination of women who are not infected with HSV can reduce the spread of HSV-1 and the risk of HSV-1-related genital herpes infection, but has no significant preventive effect on HSV-2 [44].

Reducing the efficiency of mother-to-child transmission includes reducing the risk of in utero, peripartum and postnatal virus transmission. Antiviral therapy may reduce the occurrence of genital lesions at the time of delivery, reduce the need for caesarean delivery and help to prevent postpartum infection. Since $85 \%$ of neonatal infections occur during the delivery phase, prevention should focus on avoiding exposure of newborns to genital herpes lesions and shed viruses during birth. The US CDC has suggested that all pregnant women should be asked before labor whether they have symptoms related to genital herpes (including prodromal symptoms), and should be carefully checked for herpetic skin lesions. Performing a cesarean section before rupture of the membrane is the best protection against HSV infection if genital herpetic lesions are present. If there are no genital herpes or prodromal symptoms, cesarean section is not recommended. It should be emphasized that cesarean section cannot completely block the spread of HSV, because intrauterine infection is also possible.

Antiviral therapy can effectively reduce the recurrence rate of prenatal genital herpes and the probability of inoculation during the delivery stage, and this helps to reduce the risk of mother-to-child transmission during cesarean section. However, data regarding the efficacy of antiviral therapy in reducing the incidence of neonatal infection are still lacking and further studies are needed. It is worth mentioning that in the last five years, intensive work has been carried out on the development of an HSV-2 vaccine. This should reduce HSV 2 infection and the probability of viral transmission. Such studies are currently undergoing Phase 1 and Phase 2 clinical trials [44]. $10 \%$ of HSV infections occur after birth, and may be due to transmission from a healthcare worker or family member [35]. HSV causes labial as well as genital herpes, and herpetic vesicles can appear on the hands as well. Virus can be also transmitted to newborns by caring, kissing, and touching. It is therefore recommended that mothers with known or suspected labial or genital herpes avoid close contact with newborns.

\section{Conclusions}

HSV mother-to-child transmission is uncommon, but can cause serious harm to the newborn. Because HSV prophylactic and therapeutic vaccines are still in the research stage, prevention of maternal infection, early detection and early antiviral therapy are still the main measures for the prevention of HSV mother-to-child transmission. Prevention of maternal infection should focus on the prevention of new infections in late pregnancy. Furthermore, in cases of first-episode genital herpes or recurrent episodes of genital herpes during pregnancy, standardized antiviral treatment should be initiated and caesarean section is advisable. Mothers with herpetic lesions should avoid close contact with the newborn.

\section{Acknowledgement}

This study was funded by grants from the National Natural Science Foundation of China (NSFC 81360236), Shanghai Hospital Development Center project (SHDC12014217) and 
the Shanghai Committee of Science and Technology, China (16411961500).

This publication is supported by the National Research, Development and Innovation Fund (Hungarian NRDIF) K 120206 and by the GINOP-2.3.2-15-2016-00005 project. The project is co-financed by the European Union under the European Regional Development Fund.

\section{Correspondence to}

Prof. Yeqiang Liu, PhD

Department of Dermatopathology

Shanghai Skin Disease Hospital

No.1278 Baode Road, Jingan District

Shanghai 200443, China

E-mail: lyqdoctor@163.com

\section{References}

1 James SH, Sheffield JS, Kimberlin DW. Mother-to-child transmission of herpes simplex virus. J Ped Inf Dis Soc 2014; 3: S19S23.

2 Corey L, Wald A. Maternal and neonatal HSV infections. New Engl J Med 2009; 361(14): 1376-85.

3 James SH, Kimberlin DW. Neonatal herpes simplex virus infection. Infect Dis Clin North Am 2015; 29(3): 391-400.

4 Okonko IO, Cookey TI. Seropositivity and determinants of immunoglobulin-G ( $\operatorname{lgG}$ ) antibodies against Herpes simplex virus (HSV) types -1 and -2 in pregnant women in Port Harcourt, Nigeria. Afr. Health Sci 2015; 15(3): 737-47.

5 Perti T, Nyati M, Gray G et al. Frequent genital HSV-2 shedding among women during labor in Soweto, South Africa. Infect Dis Obstet Gynecol 2014; 2014: 258291.

6 Anjulo AA, Abebe T, Hailemichael F et al. Seroprevalence and risk factors of herpes simplex virus-2 among pregnant women attending antenatal care at health facilities in Wolaita zone, Ethiopia. Virol J 2016; 13: 43.

7 Domercant JW, Louis FJ, Hulland E et al. Seroprevalence of herpes simplex virus type-2 (HSV-2) among pregnant women who participated in a national HIV surveillance activity in Haiti. BMC Infect Dis 2017; 17: 577.

8 Kim ID, Chang HS, Hwang KJ et al. Herpes simplex virus 2 infection rate and necessity of screening during pregnancy: a clinical and seroepidemiologic study. Yonsei Med J 2012; 53(2): 401-7.

9 Delaney S, Gardella C, Saracino M et al. Seroprevalence of herpes simplex virus type 1 and 2 among pregnant women, 1989-2010. JAMA 2014; 312(7): 746-8.

10 Puhakka L, Sarvikivi E, Lappalainen M et al. Decrease in seroprevalence for herpesviruses among pregnant women in Finland: cross-sectional study of three time points 1992, 2002 and 2012. Infect Dis 2016; 48(5): 1-5.

11 Woestenberg PJ, Tjhie JH, de Melker HE et al. Herpes simplex virus type 1 and type 2 in the Netherlands: seroprevalence, risk factors and changes during a 12-year period. BMC Infect Dis 2016; 16: 364 .
12 Korr G, Thamm M, Czogiel I et al. Decreasing seroprevalence of herpes simplex virus type 1 and type 2 in Germany leaves many people susceptible to genital infection: time to raise awareness and enhance control. BMC Infect Dis 2017; 17: 471.

13 Kimberlin DW. The scarlet H. J Infect Dis 2014; 1; 209(3): 315-7.

14 Nakubulwa S, Kaye DK, Bwanga F et al. Incidence and risk factors for herpes simplex virus type 2 seroconversion among pregnant women in Uganda: A prospective study. Infect Dev Ctries 2016; 10(10): 1108-15.

15 Anzivino E, Fioriti D, Mischitelli $M$ et al. Herpes simplex virus infection in pregnancy and in neonate: status of art of epidemiology, diagnosis, therapy and prevention. Virol J 2009; 6: 40.

16 Jones CA, Raynes-Greenow C, Isaacs D. Population-based surveillance of neonatal herpes simplex virus infection in Australia, 1997-2011. Clin Infect Dis 2014; 59(4): 525-31.

17 Flagg EW, Weinstock $\mathrm{H}$. Incidence of neonatal herpes simplex virus infections in the United States, 2006. Pediatrics 2011; 127(1): e1-8.

18 Sudfeld CR, Hewett PC, Abuelezam NN et al. Herpes simplex virus type 2 cross-sectional seroprevalence and the estimated rate of neonatal infections among a cohort of rural Malawian female adolescents. Sex Transm Infect 2013; 89(7): 561-7.

19 Hemelaar SJAL, Poeran J, Steegers EAP et al. Neonatal herpes infections in the Netherlands in the period 2006-2011. Matern Fetal Neonatal Med 2015; 28(8): 905-909.

20 Looker KJ, Magaret AS, May MT et al. First estimates of the global and regional incidence of neonatal herpes infection. Lancet Glob Health 2017; 5(3): e300-e309.

21 Ambroggio L, Lorch SA, Mohamad Z et al. Congenital anomalies and resource utilization in neonates infected with herpes simplex virus. Sex Transm Dis 2009; 36(11): 680-5.

22 Pena KC, Adelson ME, Mordechai E et al. Genital herpes simplex virus type 1 in women: detection in cervicovaginal specimens from gynecological practices in the United States. J Clin Microbiol 2010; 48: 150-3.

23 Brown EL, Gardella C, Malm G et al. Effect of maternal herpes simplex virus (HSV)serostatus and HSV type on risk of neonatal herpes. Acta Obstet Gynecol Scand 2007; 86(5): 523-9.

24 James SH, Kimberlin DW. Neonatal herpes simplex virus infection: epidemiology and treatment. Clin Perinatol 2015; 42 (1): 47-59, viii.

25 Kulhanjian JA, Soroush V, Au DS et al. Identification of women at unsuspected risk of primary infection with herpes simplex virus type 2 during pregnancy. N Engl J Med 1992; 326(14): 916-20.

26 Straface $G$, Selmin A, Zanardo V et al. Herpes simplex virus infection in pregnancy. Infect Dis Obstet Gynecol 2012; 2012: 385697.

27 Brown ZA, Selke S, Zeh J et al: The acquisition of herpes simplex virus during pregnancy. N Engl J Med 1997; 337: 509-15.

28 Brown ZA, Wald A, Morrow RA et al. Effect of serologic status and cesarean delivery on transmission rates of herpes simplex virus from mother to infant. JAMA, 2003; 289(2): 203-9.

29 Finger-Jardim F, Teixeira LO, de Oliveira GR et al. Herpes simplex virus: prevalence in placental tissue and incidence in neonatal cord blood samples. J Med Virol 2014; 86(3): $519-24$. 
30 Handel S, Klingler EJ, Washburn K et al. Population - based surveillance for neonatal herpes in New York City, April 2006September 2010. Sex Transm Dis 2011; 38(8): 705-11.

31 ACOG Committee on Practice Bulletins. ACOG Practice Bulletin. Clinical management guidelines for obstetrician - gynecologists. No. 82 June 2007, Management of herpes in pregnancy. Obstet Gynecol 2007; 109(6): 1489-98.

32 Marquez L, Levy ML, Munoz FM et al. A report of three cases and review of intrauterine herpes simplex virus infection. Pediatr Infect Dis J 2011; 30: 153-7.

33 Hutto C, Arvin A, Jacobs R et al. Intrauterine herpes simplex virus infections. J Pediatr 1987; 110: 97-101.

34 Curfman AL, Glissmeyer EW, Ahmad FA et al. Initial presentation of neonatal herpes simplex virus infection. J Pediatr 2016; 172: 121-126.e1.

35 Pinninti SG, Kimberlin DW. Preventing herpes simplex virus in the newborn. J Clin Perinatol 2014; 41(4): 945-55.

36 Kimberlin DW, Lin YC, Jacobs RF et al. Natural history of neonatal herpes simplex virus infections in the acyclovir era. Pediatrics 2001; 108: 223-9.

37 Patel R, Kennedy OJ, Clarke E et al. 2017 European guidelines for the management of genital herpes. Int J STD\&AIDS 2017; O: $1-14$.
38 LeGoff J, Péré H, Bélec L. Diagnosis of genital herpes simplex virus infection in the clinical laboratory. Virol J 2014; 11: 83.

39 Kimberlin DW, Baley J. Guidance on management of asymptomatic neonates born to women with active genital herpes lesions. Pediatrics 2013; 131(2): 635-45.

40 World Health Organization. WHO guidelines for the treatment of genital herpes simplex virus. Geneva, Organization, 2016

41 Workowski KA, Bolan GA. Centers for Disease Control and Prevention. Sexually transmitted diseases treatment guidelines, 2015. MMWR Recomm Rep 2015; 64(RR-03): 1-137.

42 Kimberlin DW, Lin CY, Jacobs RF et al. National Institute of Allergy and Infectious Diseases Collaborative Antiviral Study Group. Safety and efficacy of high dose intravenous acyclovir in the management of neonatal herpes simplex virus infections. Pediatrics 2001; 108(2): 230-8.

43 Pickering LK, Baker CJ, Kimberlin DW et al. 2012 Report of the Committee on Infectious Diseases. American Academy of Pediatrics; 2012. Available from https://redbook.solutions. aap.org/DocumentLibrary/RB12_interior.pdf [Last accessed January 29, 2018].

44 Gottlieb SL, Johnston C. Future prospects for new vaccines against sexually transmitted infections. Curr Opin Infect Dis 2017; 30(1): 77-86. 


\section{Query/ Note to the author:}

AQ1: Author: Please confirm that given names (red) and surnames/family names (green) have been identified correctly. 